SCIENTIFIC REPORT

\title{
Posterior capsule opacification after implantation of a hydrogel intraocular lens
}

\section{K Hayashi, H Hayashi}

Aim: To compare the degree of posterior capsule opacification (PCO) in eyes with a hydrophilic hydrogel intraocular lens (IOL) with that in eyes with a hydrophobic acrylic IOL. Methods: Ninety five patients underwent a hydrogel IOL implantation in one eye and an acrylic $\mathrm{IOL}$ implantation in the opposite eye. The PCO value of these patients was measured using the Scheimpflug videophotography system at $1,6,12,18$, and 24 months postoperatively. The rate of neodymium:YAG (Nd:YAG) laser posterior capsulotomy and visual acuity were also evaluated.

Results: The mean PCO value in the hydrogel group increased significantly $(p<0.0001)$, while that in the acrylic group did not show significant change. The PCO value in the hydrogel group was significantly greater than that in the acrylic group throughout the follow up period. Kaplan-Meier survival analysis determined that the $\mathrm{Nd}$ :YAG capsulotomy rate in the hydrogel group was significantly higher than that in the acrylic group $(p<0.0001)$. Mean visual acuity in the hydrogel group decreased significantly with time $(p<0.0001)$, and became significantly worse than that in the acrylic group at 18 and 24 months postoperatively.

Conclusion: Posterior capsule opacification in eyes with a hydrophilic hydrogel IOL is significantly more extensive than that in eyes with a hydrophobic acrylic IOL, and results in a significant impairment of visual acuity.

M any kinds of foldable intraocular lenses (IOLs) composed of various different optic materials have been developed recently. At present, the optics of most foldable IOLs are composed of hydrophobic materials such as silicone or acrylic. The only one hydrophilic IOL currently available worldwide is a hydrogel IOL (Hydroview, H60M, Bausch \& Lomb, St Louis, MO, USA). The optic of the Hydroview IOL is composed of 2-hydroxyethylmethacrylate and 6-hydroxyethylmethacrylate, and has a water content of $18 \%$. The Hydroview optic is considered to be highly biocompatibile in terms of basic parameters such as contact angle of water and interface free energy. ${ }^{1}$

However, it has been reported that this IOL has two disadvantages: marked proliferation of lens epithelial cells onto the optic surface, ${ }^{2-7}$ and an occasional deposition of calcium hydroxyapatite into the optic material. ${ }^{8-11}$ In addition, we have shown that contraction of the anterior capsule opening in the presence of the hydrogel IOL is more extensive than that with an acrylic IOL. ${ }^{12}$ Moreover, several studies reported that patients with the hydrogel IOL developed greater posterior capsule opacification (PCO) than did those having IOLs with other optic materials. ${ }^{13-15}$ However, these studies failed to show a significant difference in visual acuity impairment due to the PCO.
The objective of this study was to examine the progression of PCO in eyes after hydrogel IOL implantation, and to compare the degree of PCO with that in eyes after acrylic IOL implantation. Because the hydrogel IOL is composed of hydrophilic material, we paid particular attention to the growth pattern of PCO in eyes with a hydrogel IOL.

\section{PATIENTS AND METHODS}

All patients who were admitted consecutively to the Hayashi Eye Hospital for bilateral cataract surgery between December 1999 and June 2000 were screened for inclusion in this study. Preoperative exclusion criteria were ocular pathology other than senile cataract, history of prior ocular surgery or inflammation, eyes scheduled for extracapsular cataract extraction, a pupillary diameter less than $6.0 \mathrm{~mm}$ after full dilation, pseudoexfoliation syndrome, and diabetics and patients who could not be available for follow up. Postoperative exclusion criteria after randomisation were any surgical complication, asymmetrical or out-of-the-bag implantation, any difficulties with the analysis, and patient refusal of examination. Patient screening was continued until 100 patients who were to undergo phacoemulsification and IOL implantation were recruited.

The study protocol was approved by the Institutional Review Board and written informed consent was obtained from each patient. All patients were randomly assigned the day before surgery to one of the two groups: those who received a hydrogel IOL (Hydroview, H60M) in the left eye, and an acrylic IOL (AcrySof, MA60BM, Alcon Surgical, Fort Worth, TX, USA) in the right eye, and those who were implanted with a hydrogel IOL in the right eye and an acrylic IOL in the left eye. The controller of this clinical trial generated a randomisation code with equal numbers using random number tables, and kept concealed the assignment schedule until all data were collected to ensure allocation concealment. Patients, examiners, operating room staff who allocated the IOL to the patients, and the surgeon (who was also the data analyst) were all masked to randomisation. The H60M is a three piece hydrogel IOL, while the MA60BM is a three piece acrylic IOL. Both IOLs have a $6.0 \mathrm{~mm}$ round optic and polymethylmethacrylate (PMMA) modified C loops.

All surgeries were performed by a single surgeon $(\mathrm{KH})$ using the same surgical procedure that has been described previously. ${ }^{16}$ Firstly, a $3.5 \mathrm{~mm}$ straight scleral incision was made for IOL implantation. After incision, a continuous curvilinear capsulorrhexis, measuring approximately $5.5 \mathrm{~mm}$ in diameter, was accomplished using a bent needle. After hydrodissection, endocapsular phacoemulsification of the nucleus and aspiration of the residual cortex were carried out. Using a steel keratome, the wound was enlarged to $4.1 \mathrm{~mm}$ for IOL implantation. The lens capsule was inflated with $1 \%$ sodium hyaluronate (Healon, Pharmacia, Uppsala, Sweden), after which the IOL was placed into the capsular bag using the folding forceps. After insertion, the viscoelastic material was thoroughly evacuated. In no case was any 
suture placed. In this series, all surgeries were uneventful and the IOLs were accurately implanted in the capsular bag.

The PCO density value in these patients was measured using the Scheimpflug videophotography system (EAS-1000, NIDEK, Gamagori, Japan) at 1, 6, 12, 18, and 24 months after surgery. The PCO density value was determined using a method previously described. ${ }^{17}$ In brief, the examiner first obtained a Scheimpflug slit image of the IOL at the $0^{\circ}, 45^{\circ}$, $90^{\circ}$, and $135^{\circ}$ meridians after full dilation of the pupil. The highest quality image was transferred to an online image analysis computer. The average scattering light density of the central $3.00 \times 0.25 \mathrm{~mm}$ area of the posterior capsule, and of the central $3.00 \times 0.25 \mathrm{~mm}$ area of the anterior IOL surface was measured using the axial densitometry of the computer. The PCO density value was expressed in computer compatible tape steps (CCT): scattering light density obtained by the densitometry divided the range from 0 to 255 (256 steps). The PCO density value in one cross sectional image was determined by subtracting the scattering light density of the anterior IOL surface area from that of the posterior capsule area. The PCO values of the four meridians were then averaged and considered to represent the PCO value. In addition, the area of the anterior capsule opening was also measured using the EAS-1000 system at one week after surgery using the method previously described. ${ }^{18}$

Visual acuity and the number of eyes that required neodymium: yttrium-aluminum-garnet (Nd:YAG) laser posterior capsulotomy were also examined. Best corrected visual acuity on decimal charts was recorded at each visit and this acuity was converted to logarithm of minimal angle of resolution (logMAR) for statistical analysis. An Nd:YAG capsulotomy was performed when an eye lost two or more decimal lines of acuity or when the patient complained of blurred vision. For those patients who underwent Nd:YAG capsulotomy, the PCO value just before Nd:YAG capsulotomy was used for further statistical analysis. The rate of Nd:YAG capsulotomy was examined using Kaplan-Meier survival analysis. All measurements were performed by experienced ophthalmic technicians who were not informed of the purpose of the study.

For the continuous variables, the Mann-Whitney $U$ test was used to compare differences between the hydrogel and acrylic IOL groups. The repeated measures analysis of variance (ANOVA) was used to compare differences at the various time points. The survival curves of eyes in the two groups that required Nd:YAG capsulotomy were compared using the Mantel-Cox log rank test. Discrete variables were compared using the $\chi^{2}$ test. Any differences showing a p value of less than 0.05 were considered to be statistically significant.

\section{RESULTS}

The participant flow in this study is shown in figure 1. As the surgical procedures were the same for the two IOLs, the patients did not know the type of IOL implanted. The examiners were also unaware of the type of IOL used. Furthermore, because the controller of this trial kept concealed the assignment schedule until the end of the study, the data analyst, who was also the surgeon, did not know the type of IOL used. By 18 months after surgery, one patient died and two patients did not appear for follow up because of scheduling conflict; by 24 months after surgery, two patients did not appear for follow up because of illness. Therefore, 95 patients (95\%) completed the two year follow up.

The mean (standard deviation) age of the patients was 71.4 (SD 6.5) years, with a range of 52 to 85 years. There were 34 men and 66 women. No statistically significant difference was found between the hydrogel and acrylic IOL groups

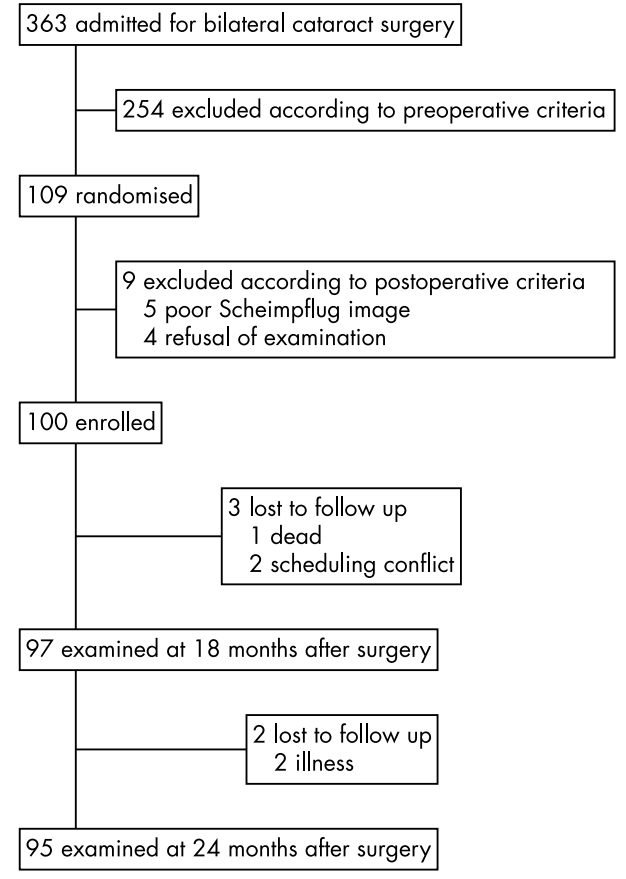

Figure 1 Chart of participant flow.

regarding the area of the capsulorrhexis opening (26.9 (SD 3.6) $\left.\mathrm{mm}^{2} v 26.3(\mathrm{SD} 3.6) \mathrm{mm}^{2}, \mathrm{p}=0.2695\right)$.

Figure 2 shows the changes in mean (SD) PCO values in the hydrogel and acrylic groups. The mean PCO value in the hydrogel group increased significantly $(p<0.0001)$, while that in the acrylic group did not show any significant change $(p=0.1127)$. More specifically, the PCO value in the hydrogel group increased in the early postoperative periods, and appeared to reach a plateau by 12 months after surgery. Furthermore, the PCO value in the hydrogel group was significantly greater than that in the acrylic group throughout the observation period.

Of the 100 eyes in each group, $28(28 \%)$ in the hydrogel group and two $(2 \%)$ in the acrylic group required Nd:YAG capsulotomy within 24 months after surgery. Kaplan-Meier survival analysis demonstrated that the rate of $\mathrm{Nd}$ :YAG

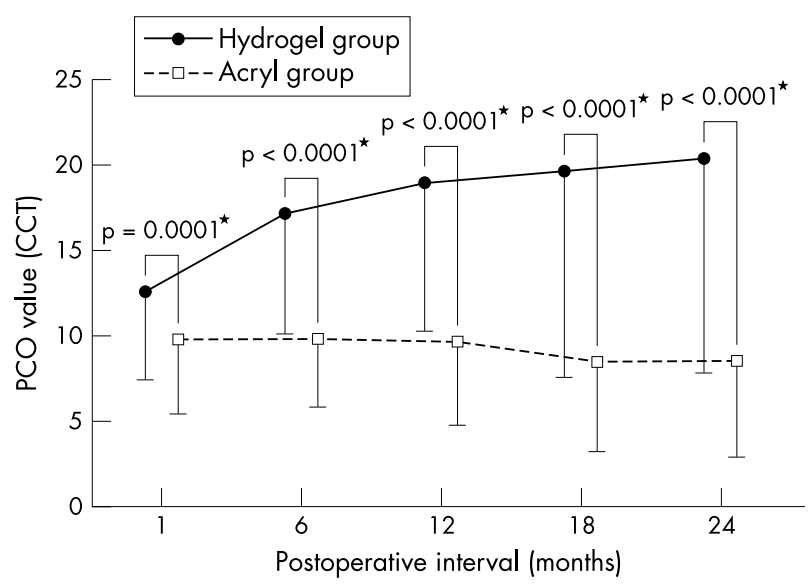

Figure 2 Changes in mean (SD) posterior capsule opacification (PCO) value in the hydrogel and acrylic IOL groups. The PCO value in the hydrogel group increased significantly, while the change in the PCO value was not significant in the acrylic group. The mean PCO value in the hydrogel $\mathrm{IOL}$ group was significantly greater than that in the acrylic group throughout follow up. 


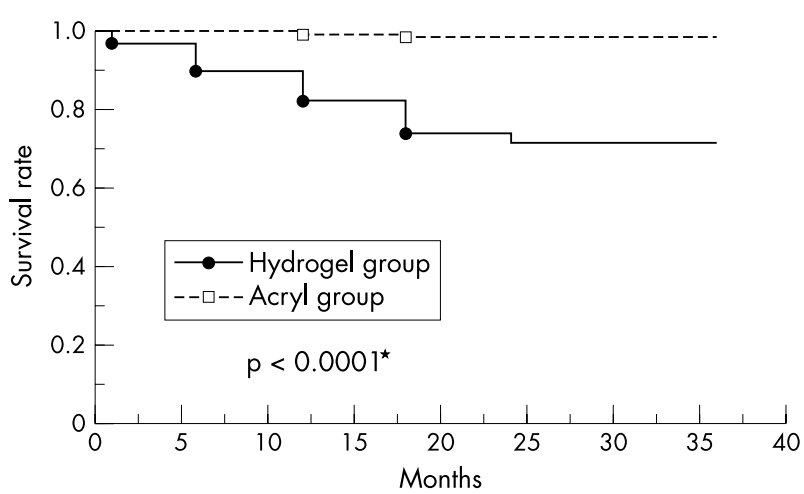

Figure 3 Kaplan-Meier survival plots of the two groups for eyes not requiring neodymium:yttrium-aluminum-garnet (Nd:YAG) laser posterior capsulotomy. The survival curve in the hydrogel group was significantly worse than that in the acrylic group $(p<0.0001$, MantelCox log rank test).

capsulotomy in the hydrogel group was significantly higher than that in the acrylic group $(\mathrm{p}<0.0001$; fig 3$)$

Figure 4 shows the mean visual acuity in the two groups. At one month after surgery, best corrected visual acuity between the two groups was almost the same $(p=0.8756)$. However, thereafter, the visual acuity in the hydrogel group worsened significantly with time $(p<0.0001)$, while the decrease in the acrylic group was not significant $(p=0.0730)$. Subsequently, at 18 and 24 months after surgery, visual acuity in the hydrogel group became significantly worse than that in the acrylic group.

Figures $5 \mathrm{~A}$ and $\mathrm{B}$ are retroillumination photographs showing bilateral eyes of a representative patient at 24 months after surgery. In the eye with a hydrogel IOL (A), fibrosis of the anterior capsule along the capsulorrhexis margin is marked. A flat proliferation of lens epithelial (fibre) cells over the posterior capsule is noted, but swelling of these cells is slight. In the opposite eye with an acrylic IOL (B), the fibrosis of the anterior capsule is slight and the posterior capsule is completely clear.

\section{DISCUSSION}

Our study quantitatively demonstrates that the degree of PCO in the eyes with a hydrogel IOL is more extensive than that in the eyes with an acrylic IOL. Specifically, PCO in the presence of a hydrogel IOL increased from the early postoperative

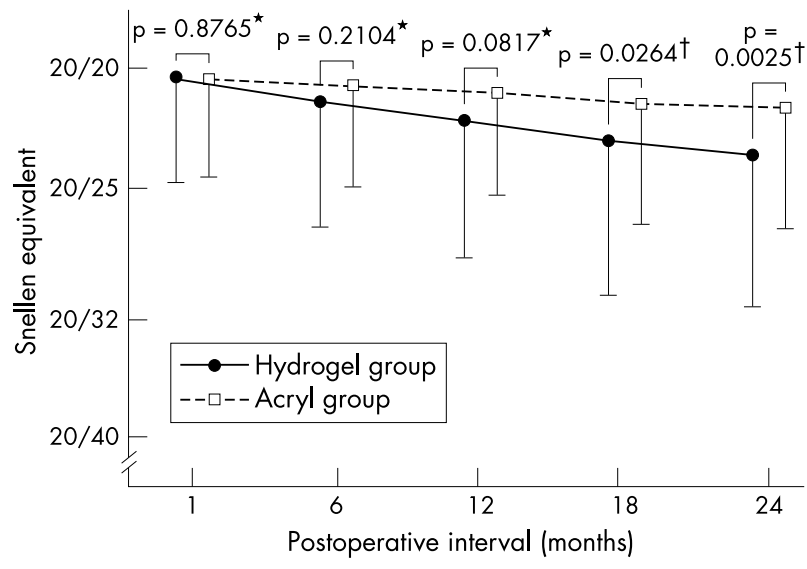

Figure 4 Changes in mean (SD) visual acuity in the hydrogel and acrylic IOL groups. Mean visual acuity in the hydrogel group was worse than that in the acrylic group; this difference was significant at 18 and 24 months after surgery.
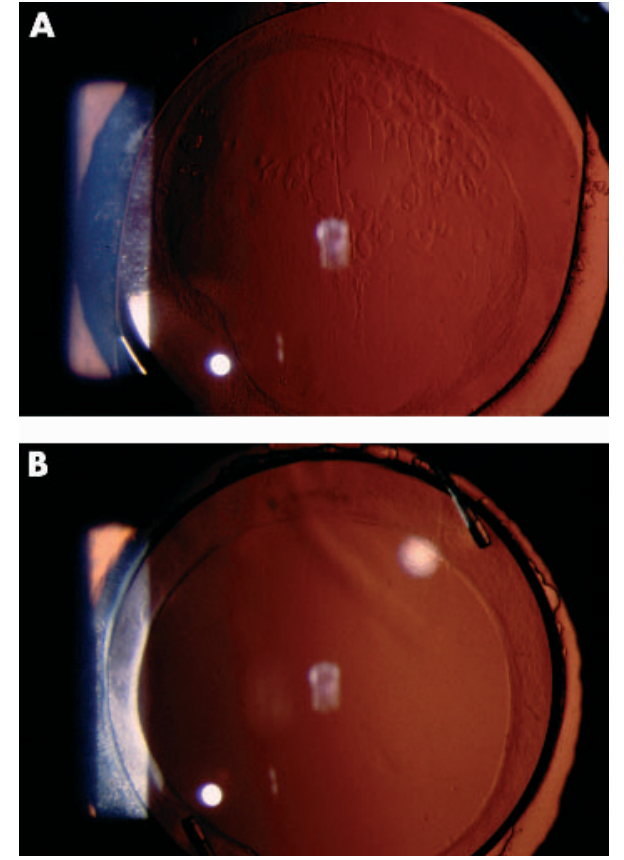

Figure 5 Retroillumination photographs showing the bilateral eyes of a representative patient at 24 months after surgery. In an eye with a hydrogel IOL (A), fibrosis of the anterior capsule along the capsulorrhexis margin is marked. A flat proliferation of lens fibre cells over the posterior capsule is noted. However, swelling of these cells is slight. In the opposite eye with an acrylic IOL (B), fibrosis of the anterior capsule is slight and the posterior capsule is completely clear.

period, but the increase virtually reached a peak by 12 months after surgery. Furthermore, the Nd:YAG capsulotomy rate was also worse with the hydrogel IOL than with the acrylic IOL; the percentage difference in the Nd:YAG capsulotomy rate between groups within two years after surgery was $26 \%$, which is considered to be clinically significant.

At one month after surgery, the visual acuity was the same between eyes with the hydrogel IOL and those with the acrylic IOL. Thereafter, however, visual acuity in eyes with the hydrogel IOL worsened significantly with time, so that the visual acuity in eyes with a hydrogel IOL was worse than that in eyes with an acrylic IOL in the later postoperative period. Thus, our results clearly show that PCO in the presence of a hydrogel IOL impairs visual acuity more so than does that in the presence of an acrylic IOL.

Previous semiquantitative studies reported that the frequency of eyes developing PCO was higher with the hydrogel IOL than that with the acrylic IOL. ${ }^{14}{ }^{15}$ Only one quantitative study, by Hollick et al, ${ }^{13}$ also revealed that the hydrogel IOL was associated with significantly greater PCO than that seen with polymethylmethacrylate and silicone IOLs. However, these studies ${ }^{13-15}$ failed to show a significant difference in visual acuity. Our study showed for the first time that the hydrogel IOL led to a significantly greater impairment of visual acuity due to PCO.

On slit lamp examination, we noted that the progression pattern of PCO with the hydrogel IOL was different from that with the other types of IOL. It is known that capsular fibrosis due to proliferation of fibroblast-like lens epithelial cells is predominant in the early postoperative period. ${ }^{19}{ }^{20}$ However, with the hydrogel IOL, flat spindle shaped lens epithelial cells invaded the retrolental space from the early period. Because these cells were well demarcated and not accompanied by fibrosis, they were considered to be lens fibre cells that might 
have originated at the lens equator. In general, the fibre cells are grown and subsequently develop into Elschnig pearls. However, with the hydrogel IOL, swelling of the fibre cells was not so marked as to form a thick layer even during the two year follow up. Thus, early invasion of a flat layer of lens fibre cells was characteristic of PCO after hydrogel IOL implantation. As the proliferation pattern of lens epithelial cells may not be influenced by the IOL design, this distinct pattern of PCO may be caused by the hydrogel optic material.

The most important characteristic of hydrogel material is certainly its hydrophilic properties, which are considered to be highly biocompatible in terms of basic parameter such as contact angle with water. However, this may lead to weak adhesion with the lens capsule, and thus allow active proliferation of lens epithelial cells in the retrolental space. Indeed, it has been experimentally confirmed that this hydrogel optic adheres very weakly to the collagen film (unpublished data). Furthermore, it is known that a membranous growth of lens epithelial cells onto the IOL surface occurs commonly. ${ }^{2-7}$ Our previous study also showed that fibrosis of the anterior capsule, particularly along the capsulorrhexis margin, was extensive. ${ }^{12}$ These results suggest that the proliferation of lens epithelial cells may be most active around the hydrogel optic and, therefore, results in greater PCO.

There is an important limitation in this study. The optic edge design of the two IOLs is not the same. The Hydroview IOL has a round optic edge, while the Acrysof IOL has a sharp edge. Experimental and clinical studies have shown that a sharp optic edge can prevent the invasion of lens epithelial cells into the retrolental space, which leads to less PCO. ${ }^{21-25}$ Therefore, greater PCO with the Hydroview IOL may be partly due to the round optic edge. However, based on the difference in growth pattern of lens epithelial cells, we consider that hydrogel material is surely associated with greater PCO.

In conclusion, the extent of PCO after hydrogel IOL implantation is substantially greater than that after hydrophobic acrylic IOL implantation. The rate of Nd:YAG capsulotomy was also higher with the hydrogel IOL than with the acrylic IOL, reflecting the fact that deterioration of visual acuity due to PCO was more pronounced in eyes with a hydrogel IOL. The results of previous and current studies suggest that hydrogel material may allow active proliferation of lens epithelial cells, possibly because of its hydrophilicity, and therefore may not be appropriate for use as an optic material. Further study is needed to examine the extent of PCO and anterior capsule fibrosis with the other types of IOL that have hydrophilic properties.

\section{Authors' affiliations \\ K Hayashi, Hayashi Eye Hospital, Fukuoka, Japan \\ H Hayashi, Department of Ophthalmology, School of Medicine, Fukuoka University, Fukuoka, Japan}

The authors have no proprietary interest in any of the materials described in this article.
Correspondence to: Dr K Hayashi, Hayashi Eye Hospital, 4-7-13 Hakataekimae, Hakata-Ku, Fukuoka 812, Japan;

hayashi-ken@hayashi.or.jp

Accepted for publication 7 June 2003

\section{REFERENCES}

1 Cunnan CM, Tarbaux NM, Knight PM. Surface properties of intraocular lens materials and their influence on in vitro cell adhesion. J Cataract Refract Surg 1991; 17:767-73.

2 Lenis K, Philipson B. Lens epithelial growth on the anterior surface of hydrogel IOLs: an in vitro study. Acta Ophthalmol Scand 1998;76:184-7.

3 Hollick EJ, Spalton DJ, Ursell PG. Surface cytologic features on intraocular lenses: can increased biocompatibility have disadvantages? Arch Ophthalmol $1999 \cdot 117 \cdot 872-8$

4 Koch MU, Kalicharan D, van der Want JJ. Lens epithelial cell layer formation related to hydrogel foldable intraocular lenses. J Cataract Refract Surg 1999;25:1637-40.

5 Goarnisson S, Hennekes R. Medium term results of HEMA intraocular lenses (hydroview ${ }^{\circledR}$ ). Bull Soc Belge Ophtalmol 1999;272:63-8.

6 Mullner-Eidenbock A, Amon M, Schaversberger J, et al. Cellular reaction on the anterior surface of 4 types of intraocular lenses. J Cataract Refract Surg $2001 ; 27: 734-40$

7 Tognetto D, Toto L, Ballone E, et al. Biocompatibility of hydrophilic intraocular lenses. J Cataract Refract Surg 2002:28:644-51.

8 Bucher PJM, Buchi ER, Daicker BC. Dystrophic calcification of an implanted hydroxyethylmethacrylate intraocular lens. Arch Ophthalmol 1995; 113:1431-5.

9 Werner L, Apple DJ, Escobar-Gomez M, et al. Postoperative deposition of calcium on the surfaces of a hydrogel intraocular lens. Ophthalmology 2000; 107:2179-85.

10 Murray RI. Two cases of late opacification of the hydroview hydrogel intraocular lens. J Cataract Refract Surg 2000;26:1272-3.

11 Yu AKF, Shek TWH. Hydroxyapatite formation on implanted hydrogel intraocular lenses. Arch Ophthalmol 2001;119:611-4.

12 Hayashi K, Hayashi H, Nakao F, et al. Anterior capsule contraction and intraocular lens decentration and tilt after hydrogel lens implantation. Br J Ophthalmol 2001:85:1294-7.

13 Hollick EJ, Spalton DJ, Ursell PG, et al. Posterior capsular opacification with hydrogel, polymethylmethacrylate, and silicone intraocular lenses: two-year results of a randomized prospective trial. Am J Ophthalmol 2000;129:577-84

14 Scaramuzza A Fernando GT, Crayford BB. Posterior capsule opacification and lens epithelial cell layer formation: Hydroview hydrogel versus AcrySof acrylic intraocular lenses. J Cataract Refract Surg 2001;27:1047-54.

15 Abela-Formanek C, Amon M, Schild G, et al. Uveal and capsular biocompatibility of hydrophilic acrylic hydrophobic acrylic, and silicone intraocular lenses. J Cataract Refract Surg 2002;28:50-61.

16 Hayashi K, Hayashi H, Nakao F, et al. Posterior capsule opacification after cataract surgery in patients with diabetes mellitus. Am J Ophthalmol 2002; 134:10-6.

17 Hayashi K, Hayashi H, Nakao F, et al. Changes in posterior capsule opacification after poly(methyl methacrylate), silicone, and acrylic intraocular lens implantation. J Cataract Refract Surg 2001;27:817-24.

18 Hayashi K, Hayashi H, Nakao F, et al. Reduction in the area of the anterior capsule opening after polymethylmethacrylate, silicone, and soft acrylic intraocular lens implantation. Am J Ophthalmol 1997;123:441-7.

19 Tan DTH, Chee SP. Early central posterior capsular fibrosis in sulcus-fixated bioconvex intraocular lenses. J Cataract Refract Surg 1993;19:471-80.

20 Hayashi K, Hayashi H, Nakao F, et al. Capsular capture of silicone intraocular lenses. J Cataract Refract Surg 1996;22:1267-71.

21 Nishi O, Nishi K. Preventing posterior capsule opacification by creating a discontinuous sharp bend in the capsule. J Cataract Refract Surg 1999;25:521-6.

22 Nishi O, Nishi K, Wickstrom K. Preventing lens epithelial cell migration using intraocular lenses with sharp rectangular edge. J Cataract Refract Surg 2000;26:1543-9.

23 Nishi O, Nishi K, Akura J. Speed of capsular bend formation at the optic edge of acrylic, silicone, and poly(methyl methacrylate) lenses. J Cataract Refract Surg 2002;28:431-7.

24 Kruger AJ, Schauersberger J, Abela C, et al. Two year results: Sharp versus rounded optic edges on silicone lenses. J Cataract Refract Surg 2000;26:566-70.

25 Buehl W, Findl O, Menapace R, et al. Effect of an acrylic intraocular lens with a sharp posterior optic edge on posterior capsule opacification. J Cataract Refract Surg 2002;28:1105-11. 